\title{
Whistler instabilities from the interplay of electron anisotropies in space plasmas: A quasilinear approach
}

\author{
S. M. Shaaban,,$^{1,2 \star}$ and M. Lazar, ${ }^{1,3}$ \\ ${ }^{1}$ Centre for Mathematical Plasma Astrophysics, KU Leuven, Celestijnenlaan 200B, B-3001 Leuven, Belgium. \\ ${ }^{2}$ Theoretical Physics Research Group, Physics Department, Faculty of Science, Mansoura University, 35516, Mansoura, Egypt. \\ ${ }^{3}$ Institut für Theoretische Physik, Lehrstuhl IV: Weltraum- und Astrophysik, Ruhr-Universität Bochum, D-44780 Bochum, Germany.
}

Accepted XXX. Received YYY; in original form ZZZ

\begin{abstract}
Recent statistical studies of observational data unveil relevant correlations between whistler fluctuations and the anisotropic electron populations present in space plasmas, e.g., solar wind and planetary magnetospheres. Locally, whistlers can be excited by two sources of free energy associated with anisotropic electrons, i.e., temperature anisotropies and beaming populations carrying the heat flux. However, these two sources of free energy and the resulting instabilities are usually studied independently preventing a realistic interpretation of their interplay. This paper presents the results of a parametric quasilinear study of the whistler instability cumulatively driven by two counter-drifting electron populations and their anisotropic temperatures. By comparison to individual regimes dominated either by beaming population or by temperature anisotropy, in a transitory regime the instability becomes highly conditioned by the effects of both these two sources of free energy. Cumulative effects stimulate the instability and enhance the resulting fluctuations, which interact with electrons and stimulate their diffusion in velocity space, leading to a faster and deeper relaxation of the beaming velocity associated with a core heating in perpendicular direction and a thermalization of the beaming electrons. In particular, the relaxation of temperature anisotropy to quasi-stable states below the thresholds conditions predicted by linear theory may explain the observations showing the accumulation of these states near the isotropy and equipartition of energy.
\end{abstract}

Key words: (Sun:) solar wind - instabilities - waves - methods: numerical

\section{INTRODUCTION}

Two prominent sources of free energy are revealed by the velocity distributions of plasma particles in space plasmas, i.e., temperature anisotropies and beaming (or strahl) populations (Pilipp et al. 1987; Crooker et al. 2003; Štverák et al. 2008; Viñas et al. 2010; Tong et al. 2019a). These anisotropies are at the origin of various instabilities enhancing the wave fluctuations and turbulence detected at kinetic scales (Sahraoui et al. 2009; Bale et al. 2009; Alexandrova et al. 2013; Wilson III et al. 2013). In collision-poor plasmas from space, the kinetic wave fluctuations play major roles, interacting resonantly with plasma particles and triggering not only their relaxation (Saito \& Gary 2007; Shaaban et al. 2016; Gershman et al. 2017; Lazar et al. 2018b; López et al. 2019; Shaaban et al. 2019a) but also the wave energy dissipation at small scales (Leamon et al. 1998; Saito et al. 2008; Parashar et al. 2009; Goldstein et al. 2015). In the present paper we investigate the whistler fluctuations (Kennel \& Petschek 1966; Gary et al. 1975; Gary 1993; Shaaban et al. 2018a; Lazar et al. 2019), also known as electromagnetic electron cyclotron modes (Cuperman 1981), and focus on the instability of

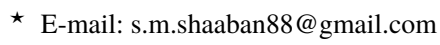

these modes, cumulatively driven by (counter-)beaming electron populations and their temperature anisotropy. In this case linear theory shows significant changes of the instability conditions and growth rates (Lazar et al. 2008; Shaaban et al. 2018a), motivating the interest for an extended quasilinear (QL) study to characterize the long-term evolution of growing fluctuations which interact with electrons and contribute to their relaxation. The observed whistler fluctuations have not only a sufficiently wide frequency width but also low amplitudes, comparing to the background magnetic field $B_{0}$, i.e., $\delta B^{2}<<B_{0}^{2}$ (Tong et al. 2019a,b), which give more credits to a $\mathrm{QL}$ approach to provide valid descriptions of these fluctuations and their action back on anisotropic electrons. A QL approach may therefore help to understand the observations of whistler like fluctuations which are often associated with combinations of counterbeaming electron populations and their temperature anisotropies, see Tong et al. (2019a,b).

The most popular is probably the whistler instability driven by the electrons with anisotropic temperature $T_{\perp}>T_{\|}$(where $\perp$ and $\|$ denote directions with respect to the magnetic field) (Gary \& Wang 1996), recent studies showing also implications in the solar wind conditions (Štverák et al. 2008; Lazar et al. 2018a; Berčič et al. 2019; Shaaban et al. 2019b). Thus, the fact that instability thresh- 
olds shape the observed temperature anisotropy of the non-drifting electron populations is an indirect proof of the constraining role that this instability may play in space plasmas (Štverák et al. 2008; Lazar et al. 2018a; Berčič et al. 2019; Shaaban et al. 2019b). QL studies and numerical simulations confirm indeed the relaxation of anisotropic electrons to the same quasi-stable states predicted by the linear thresholds (Sarfraz et al. 2016; Yoon 2017; Kim et al. 2017; Shaaban et al. 2019b). However, the highest number of solar wind data concentrate below these thresholds, at lower anisotropies, and are usually explained invoking a collisional relaxation (Salem et al. 2003; Štverák et al. 2008), although particle-particle collisions become less efficient in the solar wind with increasing the heliospheric distance (e.g., at $1 \mathrm{AU}$ and beyond).

On the other hand, the beaming or strahl population carrying the electron heat flux in the solar wind (Maksimovic et al. 2005; Pagel et al. 2007; Gurgiolo et al. 2012; Berčič et al. 2019) is often associated with the enhanced fluctuations self-generated (locally) by the so-called whistler heat flux (WHF) instability (Gary et al. 1975; Gary 1985; Shaaban et al. 2018a,b; Tong et al. 2019a; Shaaban et al. 2019a; López et al. 2019). In this case the instability conditions are markedly restrained, e.g., to low beaming velocities, and to a strahl population less dense but hotter than the main ele/ctron population (Gary 1985; Shaaban et al. 2018b). That may explain difficulties encountered in describing the long-term evolution of the WHF instability (Shaaban et al. 2019a; López et al. 2019). However, recent reports from QL analysis Shaaban et al. (2019a) and numerical simulations (López et al. 2019) have explained the saturation of WHF instability by two synchronous effects, namely, a minor relaxation of the relative drifts combined with a small temperature anisotropy $\left(T_{b, \|} \gtrsim T_{b, \perp}\right)$ effectively induced to the beaming population. The observations confirm the existence of these whistler fluctuations showing also evidences of their suppression even for a moderate temperature anisotropy (Tong et al. 2019a,b). These recent results suggest that WHF instability cannot efficiently scatter and isotropize the strahl electrons, and therefore it cannot regulate the electron heat flux in the solar wind (Shaaban et al. 2019a; López et al. 2019; Kuzichev et al. 2019). However, these results undermine long-established thoughts which invoke this instability to explain long series of observations showing the decrease of relative density and pitch-angle scattering of the electron stahl with heliospheric distance (Maksimovic et al. 2005; Pagel et al. 2007; Gurgiolo et al. 2012; Berčič et al. 2019), and also an electron heatflux below a collisional level (Bale et al. 2013), not consistent with the conventional Spitzer-Härm predictions (Spitzer \& Härm 1953). The mechanisms involving the self-induced instabilities need therefore further exploration in order to understand their implications and explain the observations. Here we assume less idealized conditions, which combine beaming electron populations with intrinsic temperature anisotropies (Viñas et al. 2010; Tong et al. 2019a) and may, thus, trigger new regimes of whistler instabilities (Lazar et al. 2008; Shaaban et al. 2018a).

Such unstable states have been described in linear theory. For instance, if whistler instability is mainly driven by temperature anisotropy of electrons (e.g., $T_{\perp}>T_{\|}$), the growth rates are inhibited by (increasing) the relative drift between electron core and beam populations (Lazar et al. 2008; Shaaban et al. 2018a). On the other hand, the influence of temperature anisotropy on the WHF instability depends on the nature of that anisotropy. Thus, linear growth rates are stimulated when beaming population (subscript b) exhibit $T_{\perp b}>T_{\| b}$, but instability is inhibited by an opposite anisotropy $T_{\| b}>T_{\perp b}$ (Shaaban et al. 2018a). However, a linear approach cannot describe more complex effects deriving from the saturation of the instability and the effects of the enhanced whistler fluctuations back on electron velocity distributions.

Here we present a QL analysis able to characterize not only the linear growth, but the long-term evolution of the whistler-like instability resulting from the interplay of (counter-)beaming electron populations and their temperature anisotropy. Our results enable to identify the contribution of these instabilities to the relaxation of electron distributions, quantifying the time variations of both these sources of free energy. In section 2 we briefly introduce the linear and QL theory of whistler instabilities for such complex conditions of plasma electrons. Numerical solutions for the WHF instability under the effects of temperature anisotropies of the beam and core populations are discussed in section 3.1. In section 3.2 we analyze the complementary regime of whistler instability mainly driven by temperature anisotropies, but under the influence of small (counter-)drifts which may alter predictions made for the temperature anisotropy limits of nondrifting plasma populations. Section 4 summarizes the results of the present study and discusses their importance, in particular, for a better understanding of the observed whistler-like fluctuations and their potential implications in the isotropization of electrons in the solar wind.

\section{QUASI-LINEAR APPROACH}

In a workframe fixed to protons the counter-moving electron populations, namely, the core (subscript "c") and the beam (subscript $" b ")$ are generically described by the velocity distribution

$f_{e}\left(v_{\perp}, v_{\|}\right)=\frac{n_{c}}{n_{0}} f_{c}\left(v_{\perp}, v_{\|}\right)+\frac{n_{b}}{n_{0}} f_{b}\left(v_{\perp}, v_{\|}\right)$,

where $n_{b} / n_{e}=\delta$ and $n_{b} / n_{e}=1-\delta$ are relative number densities and $n_{0} \equiv n_{e}=n_{c}+n_{b} \approx n_{p}$ is the total density. In order to investigate the cumulative effects of electron beams and temperature anisotropy, we assume the electron populations described by drifting bi-Maxwellian distribution functions

$f_{j}\left(v_{\perp}, v_{\|}\right)=\frac{1}{\pi^{3 / 2} \alpha_{\perp j}^{2} \alpha_{\| j}} \exp \left(-\frac{v_{\perp}^{2}}{\alpha_{\perp j}^{2}}-\frac{\left(v_{\|}-U_{j}\right)^{2}}{\alpha_{\| j}^{2}}\right)$,

with thermal velocities $\alpha_{\perp, \| j}(t)=\sqrt{2 k_{B} T_{\perp, \| j} / m_{j}}$ defined in terms of the kinetic temperature components, perpendicular $\left(T_{\perp}\right)$ and parallel $\left(T_{\|}\right)$to the stationary magnetic field $\boldsymbol{B}_{0}$. Parallel drifting velocities $U_{j}$ are conditioned by $n_{c} U_{c}+n_{b} U_{b}=0$, in order to maintain a zero net current.

For a collisionless and homogeneous electron-proton plasma, the whistler modes manifest instabilities with maximum growth rates for propagation parallel to the stationary magnetic field, i.e. $\boldsymbol{k} \times$ $\boldsymbol{B}_{0}=0$. For the parallel electromagnetic modes the instantaneous linear dispersion relation reads (Gary 1985; Shaaban et al. 2018a)

$c^{2} k^{2}=\omega^{2}+\sum_{j} \omega_{e}^{2}\left[\xi_{j} Z\left(\zeta_{j}\right)+\left(\frac{T_{\perp j}}{T_{\| j}}-1\right)\left\{1+\zeta_{j} Z\left(\zeta_{j}\right)\right\}\right]$,

where $k$ is the wave number, $\omega_{e}=\left(4 \pi n_{0} e^{2} / m_{e}\right)^{0.5}$ is the electron plasma frequency, $\omega \equiv \omega_{r}+i \gamma$ is the wave frequency (represents the complex solution of the dispersion relations), $c$ is the speed of light, $T_{\perp j}, / T_{\| j} \equiv A_{j}$ is the temperature anisotropy, $\xi_{j}=\left(\omega-k U_{j}\right) k^{-1} \alpha_{\| j}^{-1}$, and

$Z\left(\zeta_{j}\right)=\frac{1}{\sqrt{\pi}} \int_{-\infty}^{\infty} \frac{e^{-x^{2}}}{x-\zeta_{j}} d t, \mathfrak{J}\left(\zeta_{j}^{ \pm}\right)>0$ 
is the transcendental plasma dispersion function (Fried \& Conte 1961) of argument

$\zeta_{j}=\frac{\omega-\Omega_{e}-k U_{j}}{k \alpha_{\| j}}$.

In a quasi-linear (QL) formalism, the general kinetic equation for the electron distributions in the diffusion approximation takes the following form (Yoon 2017)

$$
\begin{aligned}
\frac{\partial f_{j}}{\partial t} & =\frac{i e^{2}}{4 m_{j}^{2} c^{2} v_{\perp}} \int_{-\infty}^{\infty} \frac{d k}{k}\left[\left(\omega^{*}-k v_{\|}\right) \frac{\partial}{\partial v_{\perp}}+k v_{\perp} \frac{\partial}{\partial v_{\|}}\right] \\
& \times \frac{v_{\perp} \delta B^{2}(k, \omega)}{\omega-k v_{\|}-\Omega_{j}}\left[\left(\omega-k v_{\|}\right) \frac{\partial}{\partial v_{\perp}}+k v_{\perp} \frac{\partial}{\partial v_{\|}}\right] f_{j}
\end{aligned}
$$

with the spectral wave energy of the fluctuations $\delta B^{2}$ described by the wave kinetic equation

$\frac{\partial \delta B^{2}(k)}{\partial t}=2 \gamma_{k} \delta B^{2}(k)$

where $\gamma_{k}$ is the instantaneous growth rate of whistler instabilities derived from Eq. (3). The time evolution of the macroscopic moments of the eVDFs such that the temperature components $T_{\perp, \| j}$ of beam (subscript " $j=b "$ ) and core (subscript " $j=c^{\prime \prime}$ ) and their drift velocities $U_{j}$ is then governed by the following QL kinetic equations

$$
\begin{aligned}
\frac{d T_{\perp j}}{d t} & =\frac{1}{2} \frac{\partial}{\partial t} \int d \mathbf{v} m_{j} v_{\perp}^{2} f_{j}\left(v_{\perp}, v_{\|}\right) \\
\frac{d T_{\| j}}{d t} & =\frac{\partial}{\partial t} \int d \mathbf{v} m_{j}\left(v_{\|}-U_{j}\right)^{2} f_{j}\left(v_{\perp}, v_{\|}\right) \\
\frac{d U_{j}}{d t} & =\frac{\partial}{\partial t} \int d \mathbf{v} v_{\|} f_{j}\left(v_{\perp}, v_{\|}\right)
\end{aligned}
$$

The QL approach used in the present study is based on these equations. For the sake of completeness, including full mathematical derivations of Eqs. 7, the interested reader may refer to previous studies by Moya et al. (2011), Yoon (2017), Shaaban et al. (2019a,b) and refs therein.

\section{NUMERICAL SOLUTIONS}

In this section we solve the QL equations 7 and 6 numerically for four different sets of initial macroscopic plasma parameters (at $\tau=0$ ), namely, cases $1,2,3$, and 4 .

$$
\begin{aligned}
& \text { Case } 1: A_{b}(0)=0.95,1.0,1.2, A_{c}(0)=1.0, u_{b}(0)=0.6, \\
& \text { Case } 2: A_{c}(0)=A_{b}(0)=0.95,1.0,1.2, u_{b}(0)=0.6, \\
& \text { Case } 3: u_{b}(0)=0.0,0.55,0.7, A_{b}(0)=3, A_{c}(0)=1, \\
& \text { Case } 4: u_{b}(0)=0.0,0.55,0.7, A_{b}(0)=A_{c}(0)=3,
\end{aligned}
$$

and $\delta=0.05, \beta_{b}(0)=0.4, T_{b}(0)=10 T_{c}(0), W(k)=5 \times 10^{-6}$, $u_{j}=\mu^{-0.5} U_{j} / v_{A}$, where $\mu=m_{p} / m_{e}=1836$ is the proton-electron mass ratio and $v_{A}=2 \times 10^{-4} c$ is the proton Alfvén speed. QL analysis enables us to study temporal profiles of the enhanced wave fluctuations associated with the instability saturation, as well as their back reaction on macroscopic plasma parameters such as the beam and core plasma betas $\left(\beta_{\| b, c}, \beta_{\perp b, c}\right)$, temperature anisotropies $\left(A_{b, c}\right)$, and the corresponding drift velocities $\left(u_{b, c}\right)$.

\subsection{Whistler heat-flux instability}

Here we focus on the unstable WHF solutions resulted as a cumulative effect of the relative drift velocities of the beam and core components and their temperature anisotropies. In cases 1 and 2 we adopt initial conditions favorable to WHF instability by assuming small temperature anisotropies for both the beam and core populations.

\subsubsection{Case 1 - anisotropic beam electrons, $A_{b}(0) \neq 1$}

Initial plasma parameters are carefully selected in this case, adopting relatively small anisotropies $A_{b}(0) \equiv \beta_{\perp b}(0) / \beta_{\| b}(0)=0.95,1.2$, and a small plasma beta $\beta_{\| b}(0)=0.4$ for the beam, which guarantee the dominance of WHF instability and to avoid a major effect of the temperature anisotropy driven instabilities. Thus, Figure 1 shows temporal ( $\left.\tau=\left|\Omega_{e}\right| t\right)$ evolutions of the parallel (red) and perpendicular (blue) plasma beta parameters for the the beam $\left(\beta_{\|, \perp} b\right)$ and for the core $\left(\beta_{\|, \perp c}\right)$, their drift velocities $\left(u_{b, c}\right)$, and the associated magnetic wave energy $\left(\delta B^{2} / B_{0}^{2}\right)$ for three initial conditions given by different temperature anisotropies $A_{b}(0)=0.95$ (left), 1.0 (middle), 1.2 (right). For reference to previous studies, in the middle panels we consider the case of an initially isotropic beam $\left(A_{b}(0)=1.0\right)$. The saturation of the WHF instability occurs from a concurrent effect of a relatively small relaxation of drift velocities with temperature anisotropies induced in the beam and core electrons (Shaaban et al. 2019a). For $A_{b}(0)>1.0$ the instability develops faster and magnetic wave energy reaches a maximum level almost four times higher than that obtained for beams with $A_{b}(0)=1$. The enhanced fluctuations determine in this case more pronounced effects on the electron populations, e.g., faster and deeper relaxation of the beaming velocity, and enhanced selective cooling or heating of the core and beam populations. The relaxation of the beaming velocity remains however modest, but we may expect more stable distributions after saturation. Thus, for $A_{b}(0)=1.2$ the relaxation shows a quite interesting evolution, including a significant decrease of this anisotropy reaching an isotropic state $A_{b}=1$ before changing to opposite anisotropy $A_{b}\left(t_{m}\right)<1.0$, later at $\tau=\tau_{m}$. Figure 2 displays the normalized eVDFs corresponding to the QL run in case 1 with $A_{b}(0)=1.2$ (see Figure 1), as contours in $\left(v_{\perp} / c-v_{\|} / c\right)$-velocity space (left and middle) and their parallel cuts $f_{e}\left(v_{\|} / c\right)$ (right panel) at initial $\tau=0$ (red) and $\tau=\tau_{m}$ (blue) after saturation. Shown are the following contours $10^{-3}, 5 \times 10^{-3}, 10^{-2}, 0.02,0.045$, and 0.95 . Indeed, Figure 2 indicates only a minor relaxation of the beaming velocities, see the slightly visible difference between red $(\tau=0)$ and blue $\left(\tau_{m}\right)$ dashed lines, and shows the eVDF changing the anisotropy due to thermalization in parallel direction (blue solid line in the left panel). This may explain the induced temperature anisotropy of the beam component. As one can see in the middle panel, compared to initial state the eVDF becomes less asymmetric in perpendicular direction and therefore more stable against WHF instability.

\subsubsection{Case 2 - anisotropic core electrons, $A_{c}(0) \neq 1$}

In case 2, we adopt the same initial plasma parameters as in case 1 , but with anisotropic core populations $A_{c}(0)=A_{b}(0) \neq 1$. Shaaban et al. (2018a) have shown that growth rates of the WHF instability are only slightly changed under the influence of the core anisotropies, e.g., $A_{c}(0)=0.7-1.6$, see Fig 1 therein. Here in Figure 3 we display temporal profiles of the wave energy density and the electron plasma parameters, which remain the same as in case 1 , excepting the core plasma beta $\beta_{\|, \perp c}$. In all these cases, 

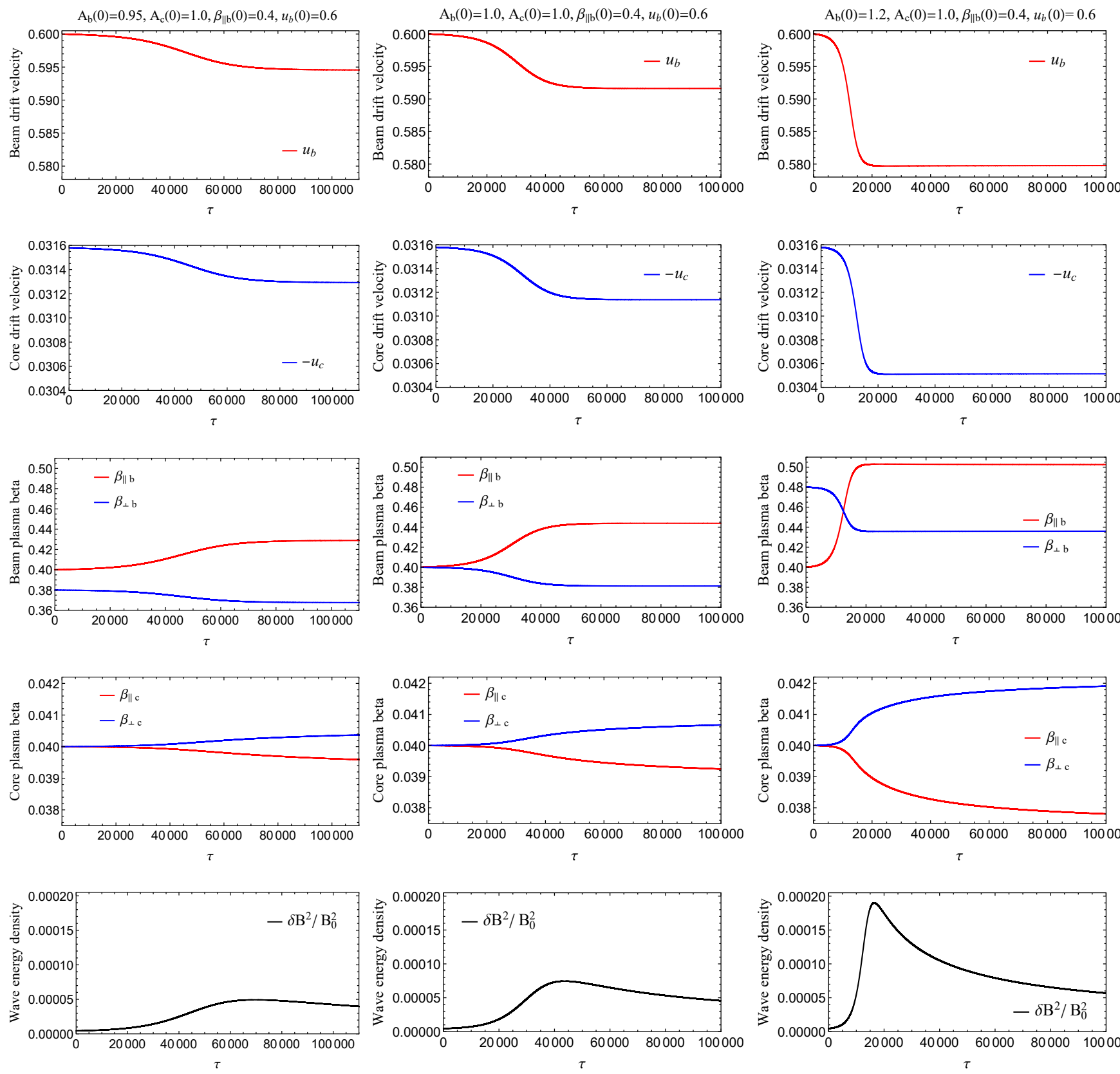

Figure 1. Case 1: Effects of the initial beam temperature anisotropies $A_{b}(0)=0.95,1,1.2$ on the instability and saturation of the whistler wave energy $\left(\delta B^{2} / B_{0}^{2}\right)$, and the relaxation of the plasma beta parameters $\left(\beta_{b, \|, \perp}\right.$ and $\left.\beta_{c, \|, \perp}\right)$, and the drift velocities $\left(u_{b, c}\right)$.

the core population gains energy (heating) in perpendicular direction, as shown by the increase of the perpendicular component of plasma beta parameter (blue), but loses energy (cooling) in parallel direction (red), see Figure 3.

\subsection{Whistler temperature anisotropy-driven instability}

In this section we consider different conditions of whistler instability (WI), as driven by sufficiently large temperature anisotropies $A_{b}(0) \equiv \beta_{b \perp}(0) / \beta_{b} \|(0)=3$.0. In addition, an initial finite drift is assumed between core and beam that may induce important effects on the enhanced wave fluctuations, inclusive on their saturation and macroscopic plasma parameters. By contrast to recent studies of WI
(Shaaban et al. 2019b), which consider only small beaming velocities, i.e. $u_{b} \leqslant 0.5$, here we assume $u_{b}(0) \geqslant 0.55$, for which the WHF mechanism is expected to contribute to the instability leading to enhanced electromagnetic fluctuations. First, in case 3, we minimize the effects of the core population, considering it isotropic, i.e., $A_{c}(0)=1.0$, in order to isolate the drift effects on the WI driven by the temperature anisotropy of beaming population. In the second part, in case 4, we examine the effects introduced by the core anisotropy $A_{c}(0)>1.0$. 

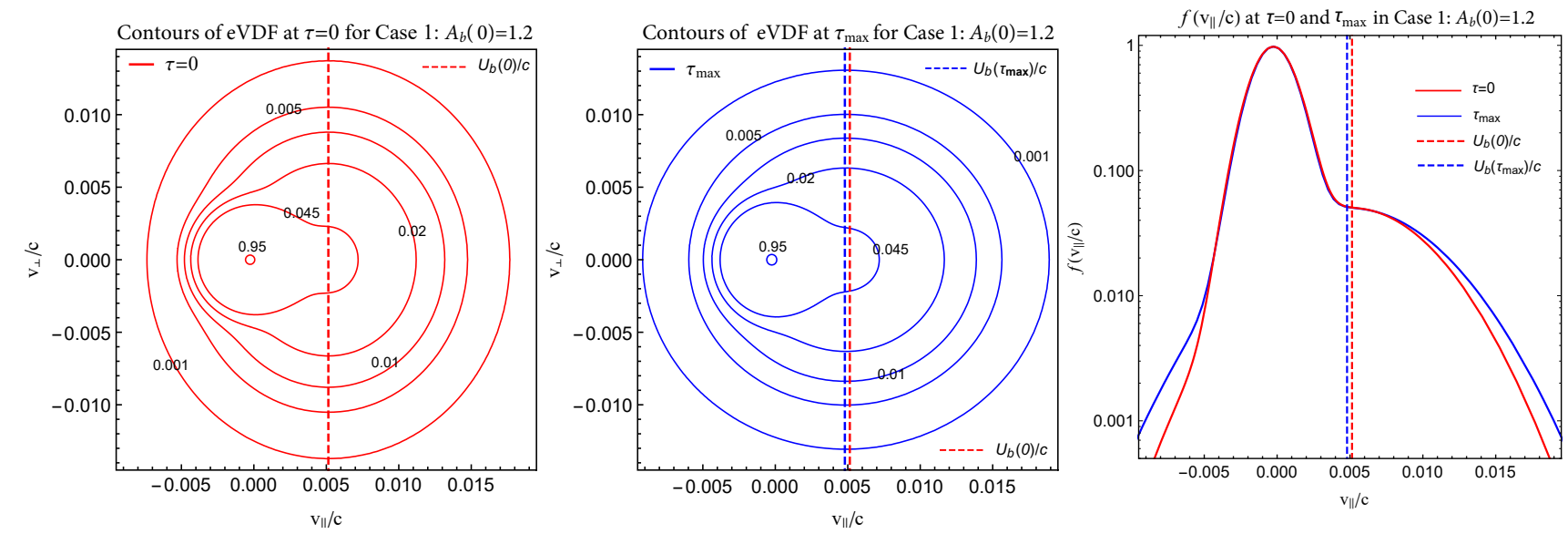

Figure 2. Contours (left and middle panels) and parallel cuts (right) of the eVDF at initial $\tau=0$ (red) and final $\tau_{m}$ (blue) stages from the same QL run for case 1 with $A_{b}(0)=1.2$ in Fig. 1 .
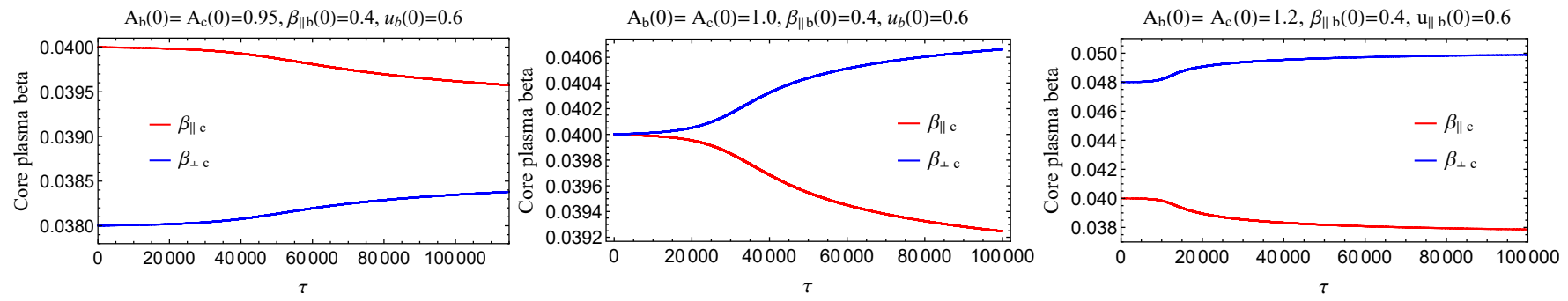

Figure 3. Case 2: The same as in Figure 1, but with initially anisotropic core $A_{c}(0)=A_{b}(0)$.

\subsubsection{Case 3 - counter-beaming electrons, $u_{c, b}(0) \neq 0$}

Fig. 4 presents temporal evolution of the initial plasma parameters in case 3 , for three different initial beaming velocities $u_{b}(0)=0.0$ (left), 0.55 (middle), 0.7 (right). For reference to previous studies, in the left panels we consider the case of an initially non-drifting electron populations with $u_{c, b}(0)=0.0$. The development of WI can regulate the initial temperature anisotropy of the beaming electrons, through the heating and cooling processes reflected by parallel (red) and perpendicular (blue) components of the electron plasma beta $\left(\beta_{\|, \perp, b}\right)$. After the saturation of WI, i.e. at $\tau_{m}$, the beam electrons are less anisotropic, i.e., $A_{b}\left(\tau_{m}\right) \gtrsim 1$, and may be at the origin of a WHF effect (Shaaban et al. 2018a). Initially isotropic $\left(A_{c}(0)=1.0\right)$ the core electrons are subjected to parallel cooling (red) and perpendicular heating (blue), as shown by the core plasma beta parameters $\left(\beta_{\|, \perp, c}\right)$, ending up with a small anisotropy induced in perpendicular direction, i.e. $A_{c}\left(\tau_{m}\right) \gtrsim 1.0$. For sufficiently large initial drifts $u_{b}(0) \geqslant 0.55$, temporal profiles of the macroscopic plasma parameters for both the beam and core populations, as well as the associated magnetic wave energy $\left(\delta B^{2} / B_{0}^{2}\right)$ are markedly changed. An increase of the initial beaming velocities $u_{b}(0) \neq 0.0$, stimulates the heating and cooling processes for both the beam and core populations leading to higher levels of magnetic wave energy after the instability saturation. For instance, an initial beaming velocity $u_{b}(0)=0.55$, increases the relaxation of the beam anisotropy, making the beam electrons isotropic $A_{b}\left(t_{m}\right)=1.0$ at later stages $\tau_{m}$. For initial beams with higher velocities, i.e., $u_{b}(0)=0.7$, their initial anisotropy $A_{b}(0)=3$ decreases passing through isotropic state $A_{b}(\tau)=1$ before reversing the anisotropy to $A_{b}\left(t_{m}\right)<1.0$, later at $\tau_{m}$. The induced temperature anisotropy reached by the core electrons $A_{c}\left(\tau_{m}\right)>1.0$ increases for initial beams with higher velocities. It is important to mention that the relaxation of the anisotropic distributions, e.g., in Figures 1 and 4, is a complex process involving a reduction of the beaming velocity but at the same time a redistribution of kinetic energy by thermalization, heating or cooling of electron populations. Instead, the magnetic wave energy increases monotonously reaching a peak of saturation, and then decreases. This dynamical variation in the wave energy may be a common feature for the long-term evolution of a multi-component plasma, not only in theory (Seough \& Yoon 2012; Kim et al. 2017; Lazar et al. 2019) but also in hybrid and PIC simulations (e.g., Shoji et al. (2009); Bortnik et al. (2011); Kim et al. (2017); Lazar et al. (2019)), when the amplitude of the developed fluctuations is small enough to prevent nonlinear effects but still allow for differential interactions with electron populations, leading in this case not only to a relaxation of beaming velocity, but also to core heating and beam thermalization. In numerical simulations the physical interpretation of this behavior is often attributed to the re-absorption of wave energy after saturation, a phase which remains weakly nonlinear or even quasilinear (QL) if well reproduced by the QL approaches (see also the explanations in Seough \& Yoon (2012), based on a competition between the damped and amplifying ranges within the integration over wave-number $k$, like in our Eq. (5)).

Based on the linear theory predictions both WHFI and WI are expected to develop with the same dispersive characteristics if the initial beaming velocity is sufficiently large $u_{b}>0.5$, and the beam temperature anisotropy is $A_{b}(0)>1.2$ (Shaaban et al. 2018a). However, linear theory cannot identify the active regimes of these 

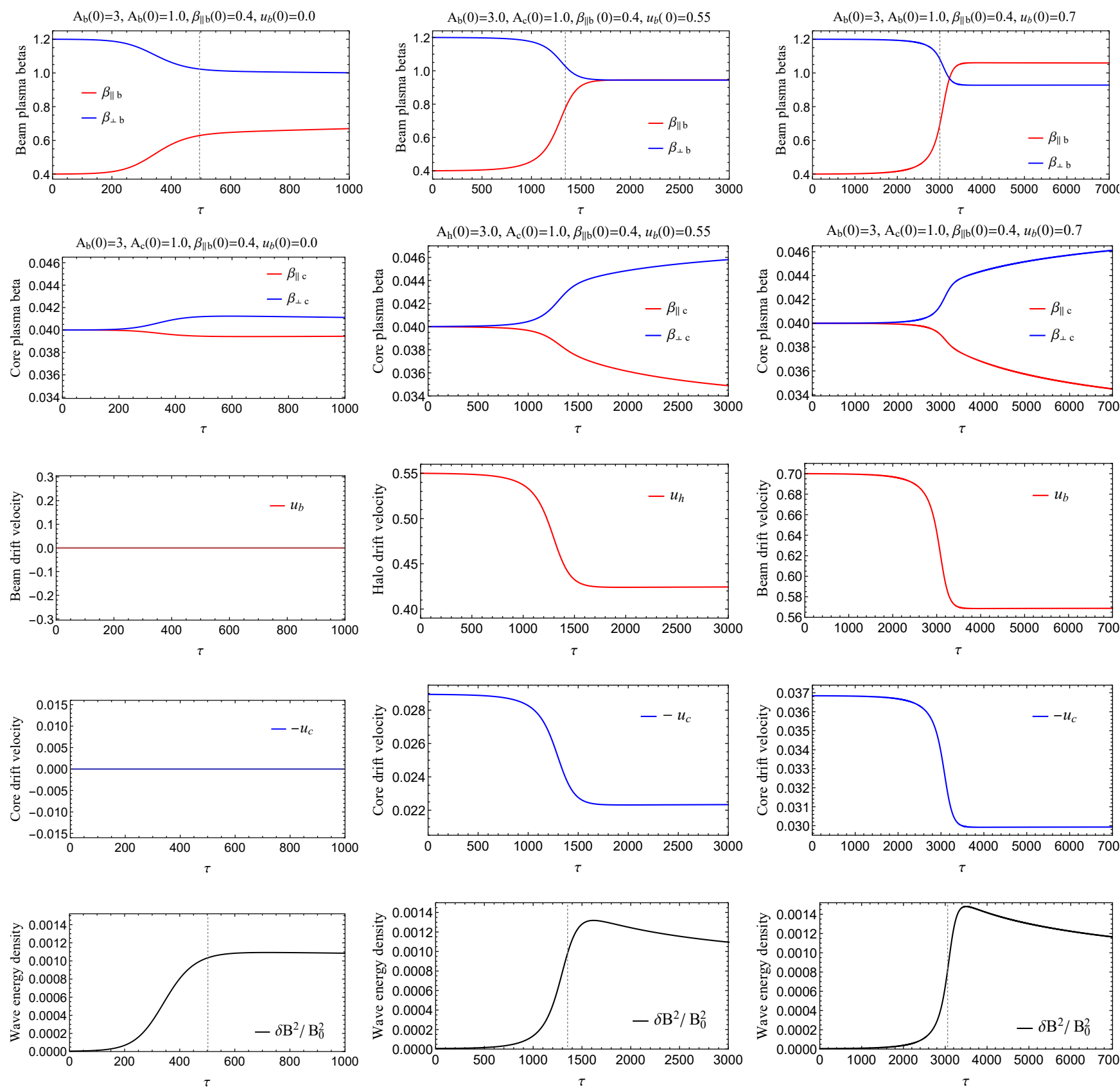

Figure 4. Case 3: Effects of the initial beam drift velocity $u_{h}(0)=0.0,0.55,0.7$ on the whistler wave energy $\left(\delta B^{2} / B_{0}^{2}\right)$, and the relaxation of the plasma parameters: plasma $\left(\beta_{b, \|, \perp}\right.$ and $\left.\beta_{c, \|, \perp}\right)$, and drift velocities $\left(u_{b, c}\right)$ of the beam and core components.

instabilities from their long-term interplay. It is a QL analysis that may provide evidences for each of these WI or WHFI regime, and enables us to understand the interplay between different degrees or sources of free energy in the plasma system. For non-drifting electron populations $u_{b, c}(0)=0.0$ (serving as a reference) the temperature anisotropy of beaming electrons $A_{b}(0) \equiv \beta_{\perp, b} / \beta_{\|, b}=$ 3.0 is partially relaxed by the enhanced fluctuations associated with the pure WI saturation, and this population ends up with lower but finite temperature anisotropy $A_{b}\left(\tau_{m}\right)=1.53$ at later stages, see left-top panel of Figure 4. Initial beaming velocities $u_{b}(0) \neq 0.0$ decelerate the relaxation process by a factor of $\sim 3$ for $u_{b}=0.55$ and $\sim 6$ for $u_{b}=0.7$ compared to nondrifting case $u_{b}=0.0$, see gray lines in the middle- and right-top panels. This delay confirms the linear theory predictions for the inhibiting effect of counterbeams on the WI (decreasing the growth rates of WI by increasing the beaming velocity), see fig. 3 in Shaaban et al. (2018a). In other words, for $u_{b}=0.55$ and $u_{b}=0.7$ the beam electrons need longer time to reach the same final anisotropy as for $u_{b}=0.0$. WHFI becomes operative and the relaxation of the beam anisotropy, as well as the wave energy density, both resemble the time evolution of a pure WHF in Figure 1 (right panels). As an evidence for the WIFI active regime, we observe the relaxation of the drift velocities for both the beam and core electrons only beyond the gray lines indicating the separation of two distinct regimes. 

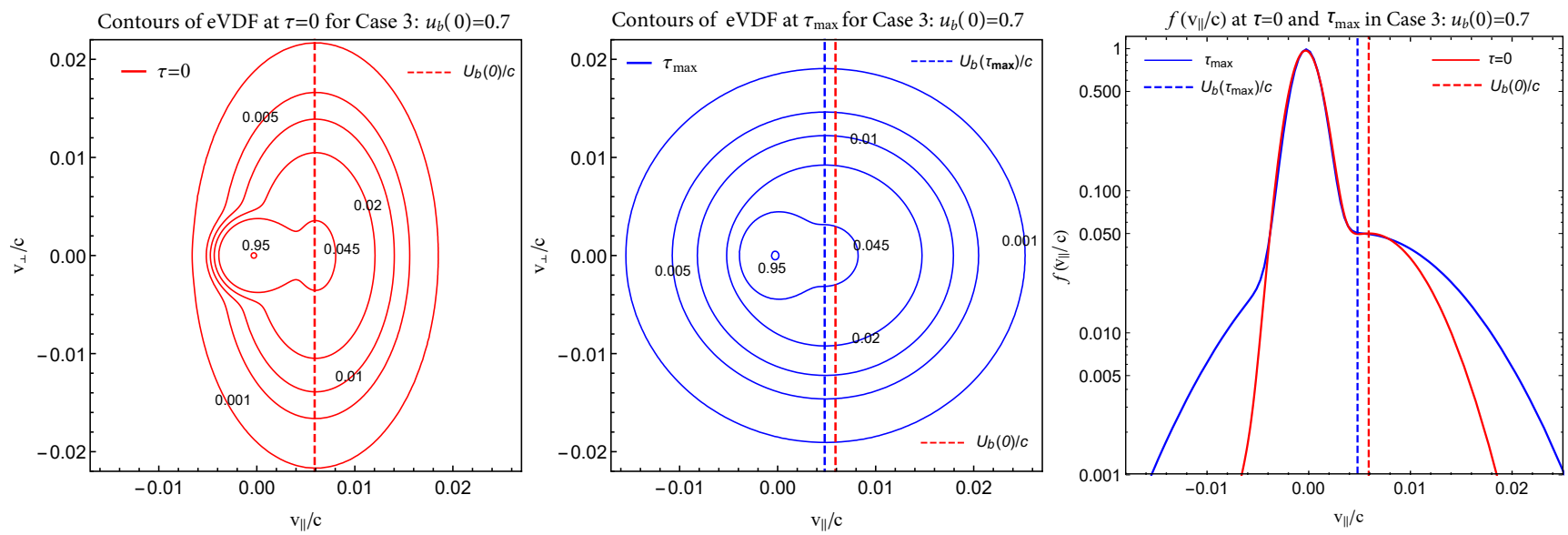

Figure 5. Contours (left and middle panels) and parallel cuts (right) of eVDFs at $\tau=0$ (red) and $\tau_{m}$ (blue) from the same QL run for case 3 with $u_{b}(0)=0.7$ in Fig. 4

Figure 5 displays contours of the eVDFs (left and middle panels) and their parallel cuts $f_{e}\left(v_{\|} / c\right)$ (right) at initial $\tau=0$ (red) and final $\tau_{m}$ (blue) stages, corresponding to the results from the QL run in case 3 with $u_{b}(0)=0.7$, see right panels in Figure 4. It is clear that after the instability saturation the eVDF is markedly different compared to the initial state, the beam electrons exhibit a small anisotropy in parallel direction and display a lower drift velocity. As a result, the eVDF at $\tau_{m}$ is less asymmetric and more stable against whistler instabilities.

\subsubsection{Case 4 - drifting anisotropic core and beam electrons}

Figure 6 describes temporal evolution of the same initial plasma parameters assumed in case 3 , but under the influence of an initially anisotropic core with $A_{c}(0)=A_{b}(0)=3$, which we name case 4 . Again, we observe a reduction of temperature anisotropies, as well as drift velocities, but within a faster evolution explained by the higher levels of wave energy density $\left(\delta B / B_{0}^{2}\right)$. Initially anisotropic, the core electrons have direct consequences on how the drift velocity affects the temporal profiles of the plasma parameters, contrasting, for instance, with the results in Figure 5. The core electrons, initially with $A_{c}(0)=3.0$, determine a lower effectiveness of the drift velocities on the resulting instability, e.g., in the relaxation of temperature anisotropy, but may boost the relaxation of the beam and core drift velocities. These results are consistent with predictions from linear theory that shows similar cumulative effects, i.e., markedly enhanced growth-rates, from the interplay of the core and beam anisotropies and their relative drifts (Lazar et al. 2018a; Shaaban et al. 2019b). The same growth rates of the WI are stimulated by the drift velocities, while the WHFI is only slightly enhanced by the core anisotropy (Shaaban et al. 2018a).

\subsubsection{Electron temperature isotropization in the solar wind}

Fig. 7 displays contours of temperature anisotropy $\left(A_{j}\right)$ thresholds derived for finite maximum growth rates $\gamma_{m} /\left|\Omega_{e}\right|=10^{-3}$, close to the marginal condition of stability (i.e. $\gamma \rightarrow 0$ ), and in terms of parallel plasma beta $\tilde{\beta}_{\| j} \equiv n_{j} \beta_{\| j} / n_{0}$. In order to confirm the constraining effect on plasma particles these thresholds are usually compared with the limits of temperature anisotropy measured in space plasmas at time scales larger than those characteristic to kinetic instabilities ( ̌̌tverák et al. 2008; Lazar et al. 2017; Shaaban et al. 2019b,c; Berčič et al. 2019). Here we refer only to beaming electrons (i.e., $j=b$ ), which are less dense but hotter than the core, and therefore more susceptible to deviations from isotropy. Contours of WI thresholds are fitted to an inverse power-law (Lazar et al. 2015; Shaaban et al. 2019b)

$A_{b}=1+\frac{a}{\tilde{\beta}_{\| b}^{d}}\left(1-\frac{r}{\tilde{\beta}_{\| b}^{s}}\right)$,

conditioning the anisotropy of the beam population $\left(A_{b}\right)$ of the corresponding plasma beta $\left(\tilde{\beta}_{\| b} \equiv n_{b} \beta_{\| b} / n_{0}\right)$. Fitting parameters $a$, $d, r$, and $s$ tabulated in Table 1 . These thresholds offer a plus of information regarding the variation of the instability growth-rates with different plasma parameters, in this case not only as a function of $\tilde{\beta}_{\| b}$, but also as a function of the core parameters, and the relative beaming velocity $\left(u_{b}\right)$. Parametrization used in the present analysis is inspired from the observations in the solar wind (Maksimovic et al. 2005; Štverák et al. 2008; Pulupa et al. 2014; Tong et al. 2019a; Berčič et al. 2019), where slow winds are usually associated with lower beaming velocities, e.g., $u_{b} \simeq 0.0$, and more energetic events like fast winds or coronal mass ejections may be characterized by higher beaming velocities $u_{b}=0.55,0.70$. In the present notation, beaming velocity $u_{b}=0.7$ implies $U_{b} \approx 30 v_{A}$ and $U_{c} \approx 1.6 v_{A}$. Two panels in Figure 7 correspond to different initial conditions, respectively, to $A_{c}(0)=1.0$ (top) and $A_{c}(0)=A_{b}(0)=3.0$ (bottom). The anisotropy thresholds of WI show a uniform variation increasing with $u_{b}$ and confirming the inhibiting effects of the relative drift (Shaaban et al. 2018a). For anisotropies $A_{b}<1$, in the bottom-right corner, we show the threshold $\left(\gamma_{m} / \Omega_{p}=10^{-2}\right)$ of the parallel firehose instability (Shaaban et al. 2019c), as an indication for the lower limits of anisotropy expected from the observations.

However, the instability thresholds predicted by linear theory may have a reduced relevance for instabilities resulted from the interplay of two plasma components (Shaaban et al. 2019a; López et al. 2019), as also in our present case. Such that, for the sake of completeness, in Figure 7 we have added the results from our QL analysis, by considering 18 different combinations of initial conditions, implying different temperature anisotropies and parallel plasma betas. The initial conditions in cases 3 and 4 are multiplied by three different initial plasma betas $\beta_{\| b}(0)=0.4,1.0$ and 3.0 (im- 

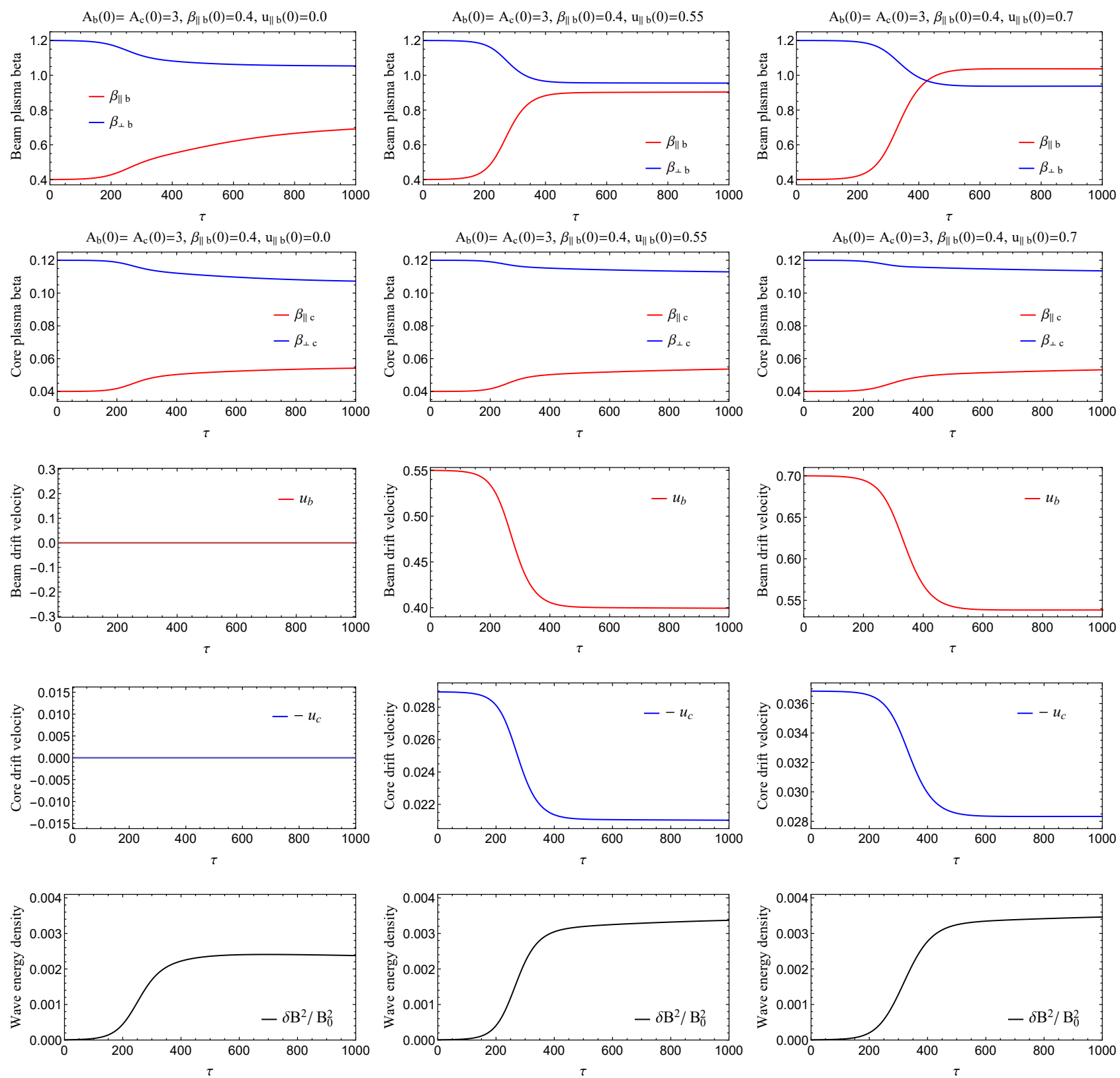

Figure 6. Case 4: The same as in Figure 3, but with initially anisotropic core $A_{c}(0)=A_{b}(0)=3.0$.

plying $\left.\tilde{\beta}_{\| b}=0.02,0.05,0.15\right)$. QL evolutions are shown as dynamical paths starting from initial positions, marked with purple filled circles, and ending after the instability saturation at final positions, shown with blue, green, and red filled circles corresponding to the initial drift velocities $u_{b}(0)=0.0,0.55$, and 0.7 . The wave energy density $\left(\delta B^{2} / B_{0}^{2}\right)$ is coded with a rainbow color scheme. In the top panel the core electrons are considered initially isotropic, in order to isolate the effects of drift velocities on the dynamical paths of temperature anisotropy. For $u_{b}=0.0$ final positions align perfectly to the corresponding anisotropy threshold predicted for WI (blue solid line) by the linear theory. For the other cases of finite $u_{b}(0)=0.55$ and 0.7 , the initially counter-streaming electron components may dramatically change the dynamical paths, which become longer to markedly lower temperature anisotropies approaching the isotropy $A_{b}=1$, or even below, changing to the opposite regime of $A_{b} \lesssim 1$.
With increasing parallel plasma beta the dynamical paths become longer for $u=0.0$, while shorter for higher $u_{b}=0.55$ or 0.7 .

Bottom panel in Figure 7 show the case of an initially anisotropic core with $A_{c}(0)=3$. For the sake of comparison, with smaller circles filled in gray, white, and black we also show the final states obtained in top panel. Again, dynamical paths for $u_{b}=0.0$ end up exactly on the corresponding WI threshold predicted by linear theory, but for $u_{b} \neq 0.0$ the final states may slightly change, remaining however much below the corresponding linear thresholds and approaching a quasi-stable regime closer to $A_{b} \sim 1.0$. To be more exact, we can specify that for $u_{b} \neq 0.0$, the temperature anisotropy of the core population, $A_{c}(0)=A_{b}(0)=3.0$, determines slightly shorter dynamical paths, in agreement with the results in Figure 6. One possible explanation already mentioned in the previous sections is given by the interplay of different sources of free energies, which may trigger different instability mechanisms, 

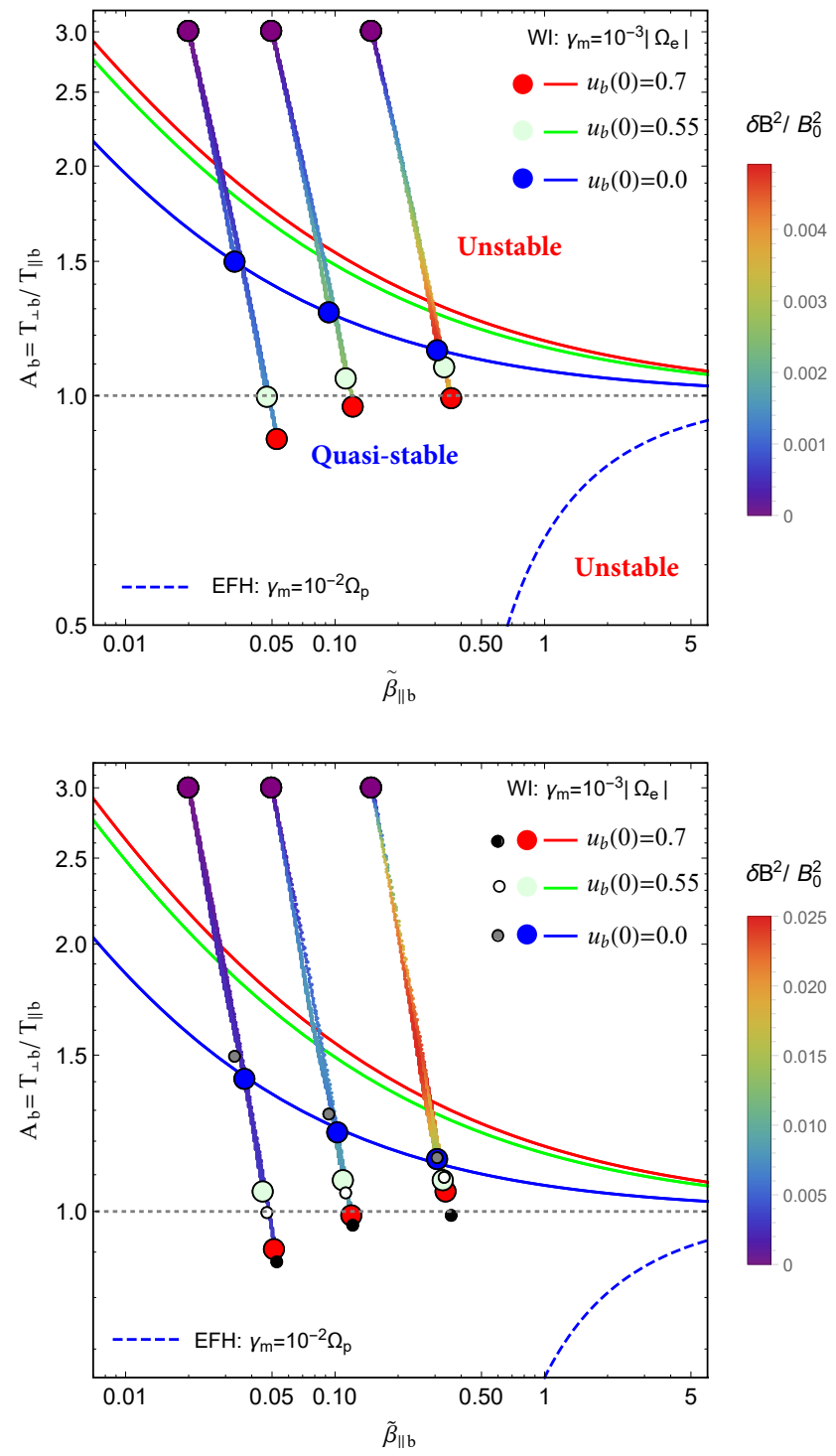

Figure 7. Dynamical paths in parametric space $\left(A_{b}, \tilde{\beta}_{\|} b\right)$ for beaming electrons for two initial conditions, $A_{c}(0)=1.0$ (top) and $A_{c}(0)=A_{b}(0)$ (bottom). Initial conditions are shown with purple filled circles, while final states are indicated with blue, green, and red filled circles. The magnetic wave energy density is color-coded. Contours corresponding to the anisotropy thresholds of WI are plotted for different beaming velocities $u_{b}=0.7$ (red), 0.55 (green), 0.0 (blue). Serving as a visual guidance, the threshold condition for the EFH instability (blue-dashed line) is taken from Shaaban et al. (2019c) for non-drifting electron populations.

i.e., WI or/and WHF, of the same branch of whistler modes (same dispersive characteristics). Their cumulative effects may lead to enhanced fluctuations and more efficient effects of these fluctuations on the relaxation of anisotropic electrons.

These results may therefore offer a valuable alternative explanation for the observations of electron populations in the solar wind, which show the most stable states accumulating (highest number of events) nearly isotropic temperatures $(A \sim 1)$ (Štverák et al. 2008; Lazar et al. 2017). The relative drift between different electron populations may exist even in the slow winds, and may therefore play a certain role in this case. Even the high-density core in the central population (subscript $j=c$ ) may respond to kinetic instabilities, which constrain their large deviations from isotropy (Štverák et al. 2008; Lazar et al. 2017). In this case the accumulation of quasistable states close to isotropy and equipartition of energy is usually attributed to another cumulative effect of binary collisions at lower heliospheric distances (e.g., in the outer corona), quantified by the so-called collisional age of plasma particles (Salem et al. 2003; Štverák et al. 2008). However, the electrons are much lighter and much faster than protons, and an explanation of their dynamics may not directly rely on the expansion and transport of the solar wind.

\section{CONCLUSIONS}

Counter-beaming populations of electrons are ubiquitous in the solar wind, and their interplay with the temperature anisotropies cannot be ignored (Štverák et al. 2008; Viñas et al. 2010; Tong et al. 2019a). Recent studies show that linear properties of whistler instabilities (i.e., WI and WHF, see above) are markedly affected the interplay of these sources of free energy (Shaaban et al. 2018a; Tong et al. 2019a). However, a linear approach is limited and cannot distinguish between different mechanisms responsible for instability or stabilization of enhanced fluctuations of the same plasma modes. We have carried out an advanced QL study of the whistler instabilities based on a parametric numerical analysis describing such complex (but less idealized) conditions resembling solar wind electron observations. Contrasting to previous studies, which restricted only to simplified approaches, e.g., (counter-)beaming Maxwellian populations with isotropic temperatures (Shaaban et al. 2019a; López et al. 2019) or non-drifting populations with anisotropic temperatures (Sarfraz et al. 2016; Lazar et al. 2018a), our results describe the long-term evolution of whistler instability under the cumulative effects of both these sources of free energy. In this case we can talk about more than two distinct regimes of the unstable fluctuations, the one controlled mostly by the electron strahl (or electron heat flux) beam, the complementary regime dominated by the temperature anisotropy of beaming electrons, and an intermediary regime. A cumulative or intermediary regime can be identified when destabilizing effects of electron beam and of (small) temperature anisotropies are comparable, leading to highly stimulated fluctuations, and implicitly to a more efficient relaxation of the beaming electrons. Thus, our present QL analysis may provide a more realistic perspective enabling to understand more complex mechanisms responsible for the whistler fluctuations and their implications in the observations, see for instance Tong et al. (2019a,b).

In section 3.1 we have adopted two sets of initial plasma parameters, i.e. cases 1 and 2, both favorable to the WHF instability. For an electron beam hotter but less dense than the core and with drift velocity lower than thermal speed the WHF instability can be self-generated (Gary et al. 1975; Gary 1985; Shaaban et al. 2018a). The QL temporal evolution of the wave energy density $\left(\delta B^{2} / B_{0}^{2}\right)$ and plasma parameters (core and beam drift velocities $u_{c, b}$, and beta parameters $\beta_{\perp j}, \beta_{\| j}$ ) are highly conditioned by the initial anisotropy of the beam population (Figure 1). A small (initial) anisotropy in perpendicular direction, i.e. $A_{b}(0)=1.2$, stimulates the instability and the resulting wave energy density, which determines a faster and deeper relaxation of the drift velocity, and induces a higher anisotropy to the core $\left(A_{c}>1\right)$. Parallel thermalization of the electron beam becomes also more pronounced leading to a complete relaxation of temperature anisotropy $\left(A_{b}=1\right)$, and later to a flip to opposite anisotropies $\left(A_{b}<1\right.$ or $\left.\beta_{\perp, b}<\beta_{\|, b}\right)$. If present, the initial anisotropy of the core has only a minor influence on the WHF 
Table 1. Fitting parameters in Eq. (8) for WI with $\gamma_{m}=10^{-3}\left|\Omega_{e}\right|$

\begin{tabular}{lcccccc}
\hline & \multicolumn{2}{c}{$u_{b}(0)=0.0$} & \multicolumn{2}{c}{$u_{b}(0)=0.55$} & \multicolumn{2}{c}{$u_{b}(0)=0.7$} \\
$A_{c}(0)$ & 3 & 1 & 3 & 1 & 3 & 1 \\
\hline$a$ & 0.07 & 0.078 & 0.16 & 0.157 & 0.18 & 0.18 \\
$d$ & 0.54 & 0.54 & 0.48 & 0.49 & 0.47 & 0.48 \\
$r / 10^{-5}$ & 1 & 2 & 2 & 3 & 1 & 2 \\
$s$ & 1.0 & 1.0 & 1.0 & 1.0 & 1.0 & 1.0 \\
\hline
\end{tabular}

fluctuations, including their saturation and the variation of plasma parameters (Figure 3).

Section 3.2 investigates plasma conditions more favorable to WI, mainly driven by the temperature anisotropy, e.g., cases 3 and 4 . Initially isotropic $\left(A_{c}(0)=1.0\right.$, case 3$)$, the core electrons gain energy from the enhanced WI fluctuations, and become anisotropic in perpendicular direction at later stages, i.e., $A_{c}\left(\tau_{m}\right)>1.0$. An initial relative drift $u_{b} \neq 0$ is another key factor that may stimulate the enhanced fluctuations and implicitly the relaxation of the anisotropy through pitch-angle scattering leading to effective perpendicular cooling and parallel heating of beaming electrons. The relaxation of temperature anisotropy becomes more pronounced in this case (Figure 4$)$, and for more energetic beams $\left(u_{b}=0.7\right)$ may end up with an opposite anisotropy after saturation. Temporal evolutions of the enhanced fluctuations and the macroscopic plasma parameters suggest transitions from one regime to another. For $u_{b}(0) \geqslant 0.55$, at early stages dominant is WI and the beam anisotropy is partially relaxed $\left(A_{b}(0)>A_{b}(\tau)>1\right)$ by the enhanced WI fluctuations, while at later stages WI is saturated and apparently the WHF instability becomes operative, leading to a complete relaxation of the temperature anisotropy $\left(A_{b}\left(\tau_{m}\right) \lesssim 1\right)$. An initially anisotropic core $A_{c}(0)=A_{b}(0)=3.0$ (case 4$)$ reduces the instability effect of beaming electrons, but may increase the level of the fluctuating magnetic field, confirming a cumulative WI of the core and beam anisotropies predicted by linear theory.

As another related application of our QL approach, in section 3.2.3 we have considered the problem of temperature isotropization in the solar wind. In the observations of electron populations the highest number of events accumulate at the quasi-stable states nearly isotropic temperatures $(A \sim 1)$, and below the instability thresholds predicted by linear theory (Štverák et al. 2008; Lazar et al. 2017). Figures 7 compares these thresholds derived from linear theory and dynamical paths of the beam anisotropy from our QL computations. For non-drifting electron populations $u_{j}(0)=0$ the final positions of the dynamical paths settle down, exactly on the corresponding anisotropy thresholds, while for $u_{j}(0) \neq 0$ the dynamical paths extend below the linear anisotropy thresholds to very small anisotropies which approach the isotropy conditions $A_{b} \lesssim 1$. To conclude, our present findings suggest that whistler instabilities cumulatively driven by multiple sources of free energy are expected to contribute to a more efficient relaxation of the anisotropic electrons, and may therefore provide a valuable alternative explanation for the isotropization of the solar wind electrons.

\section{ACKNOWLEDGEMENTS}

These results were obtained in the framework of the projects SCHL 201/35-1 (DFG-German Research Foundation), GOA/ 2015-014 (KU Leuven), G0A2316N (FWO-Vlaanderen). S.M. Shaaban acknowledges support by a FWO Postdoctoral Fellowship (Grant No. $12 \mathrm{Z} 6218 \mathrm{~N})$. Thanks are also due to Rodrigo A. López and Pe- ter $\mathrm{H}$. Yoon for insightful discussions in the framework of our joint research projects (Grant No. SF/17/007-2018, ISSI project on Kappa Distributions 2017-2019).

\section{REFERENCES}

Alexandrova O., Chen C. H., Sorriso-Valvo L., Horbury T. S., Bale S. D., 2013, Space Science Reviews, 178, 101

Bale S. D., Kasper J. C., Howes G. G., Quataert E., Salem C., Sundkvist D., 2009, Physical Review Letters, 103, 1

Bale S. D., Pulupa M., Salem C., Chen C. H. K., Quataert E., 2013, ApJL, 769,2

Berčič L., Maksimović M., Landi S., Matteini L., 2019, MNRAS, 486, 3404

Bortnik J., Omidi N., Chen L., Thorne R. M., Horne R. B., 2011, Journal of Geophysical Research: Space Physics, 116

Crooker N. U., Larson D. E., Kahler S. W., Lamassa S. M., Spence H. E., 2003, Geophys. Res. Lett., 30, 1619

Cuperman S., 1981, Reviews of Geophysics, 19, 307

Fried B., Conte S., 1961, The Plasma Dispersion Function. New York: Academic Press, doi:10.1016/B978-1-4832-2929-4.50005-8

Gary S. P., 1985, J. Geophys. Res., 90, 10815

Gary S. P., 1993, Theory of space plasma microinstabilities. Cambridge University Press, Cambridge, doi:10.1017/CBO9780511551512

Gary S. P., Wang J., 1996, J. Geophys. Res., 101, 10749

Gary S. P., Feldman W. C., Forslund D. W., Montgomery M. D., 1975, Geophys. Res. Lett., 2, 79

Gershman D. J., et al., 2017, Nat. Commun., 8, 14719

Goldstein M., Wicks R., Perri S., Sahraoui F., 2015, Phil. Trans. R. Soc. A, 373,20140147

Gurgiolo C., Goldstein M. L., Viñas A. F., Fazakerley A. N., 2012, Annales Geophysicae, 30, 163

Kennel C. F., Petschek H., 1966, J. Geophys. Res., 71, 1

Kim H. P., Hwang J., Seough J. J., Yoon P. H., 2017, Journal of Geophysical Research: Space Physics, 122, 4410

Kuzichev I. V., Vasko I. Y., Soto-Chavez A. R., Tong Y., Artemyev A. V., Bale S. D., Spitkovsky A., 2019, The Astrophysical Journal, 882, 81

Lazar M., Schlickeiser R., Poedts S., Tautz R. C., 2008, MNRAS, 390, 168

Lazar M., Poedts S., Schlickeiser R., Dumitrache C., 2015, MNRAS, 446, 3022

Lazar M., Shaaban S. M., Poedts S., Štverák 2017, MNRAS, 464, 564

Lazar M., Shaaban S. M., Fichtner H., Poedts S., 2018a, PhPl, 25

Lazar M., Yoon P. H., López R. A., Moya P. S., 2018b, J. Geophys. Res., 123,6

Lazar M., López R. A., Shaaban S. M., Poedts S., Fichtner H., 2019, Astrophysics and Space Science, 364, 171

Leamon R. J., Smith C. W., Ness N. F., Matthaeus W. H., Wong H. K., 1998, J. Geophys. Res., 103, 4775

López R. A., Shaaban S. M., Lazar M., Poedts S., Yoon P. H., Micera A., Lapenta G., 2019, ApJL, 882, L8

Maksimovic M., et al., 2005, J. Geophys. Res., 110, 1

Moya P. S., Muñoz V., Rogan J., Valdivia J. A., 2011, Journal of Atmospheric and Solar-Terrestrial Physics, 73, 1390

Pagel C., Gary S. P., de Koning C. A., Skoug R. M., Steinberg J. T., 2007, J. Geophys. Res., 112, A04103

Parashar T. N., Shay M. A., Cassak P. A., Matthaeus W. H., 2009, PhPl, 16, 032310

Pilipp W. G., Miggenrieder H., MÃijhlhÃduser K. H., Rosenbauer H., Schwenn R., Neubauer F. M., 1987, J. Geophys. Res., 92, 1103

Pulupa M. P., Bale S. D., Salem C., Horaites K., 2014, J. Geophys. Res., 119,647

Sahraoui F., Goldstein M. L., Robert P., Khotyaintsev Y. V., 2009, Phys. Rev. Lett., 102, 231102

Saito S., Gary S. P., 2007, J. Geophys. Res., 112, A06116

Saito S., Gary S. P., Li H., Narita Y., 2008, PhPl, 15, 102305

Salem C., Hubert D., Lacombe C., Bale S. D., Mangeney A., Larson D. E., Lin R. P., 2003, ApJ, 585, 1147 
Sarfraz M., Saeed S., Yoon P., Abbas G., Shah H., 2016, J. Geophys. Res., 121,9356

Seough J., Yoon P. H., 2012, Journal of Geophysical Research: Space Physics, 117, A08101

Shaaban S. M., Lazar M., Poedts S., Elhanbaly A., 2016, Astrophysics and Space Science, 361, 193

Shaaban S. M., Lazar M., Yoon P. H., Poedts S., 2018a, PhPl, 25, 082105

Shaaban S. M., Lazar M., Poedts S., 2018b, MNRAS, 480, 310

Shaaban S. M., Lazar M., Yoon P. H., Poedts S., López R. A., 2019a, MNRAS, 486, 4498

Shaaban S. M., Lazar M., Yoon P. H., Poedts S., 2019b, Astronomy \& Astrophysics, 627, A76

Shaaban S. M., Lazar M., Yoon P. H., Poedts S., 2019c, ApJ, 871, 237

Shoji M., Omura Y., Tsurutani B. T., Verkhoglyadova O. P., Lembege B., 2009, Journal of Geophysical Research: Space Physics, 114, A10203

Spitzer L., Härm R., 1953, Phys. Rev., 89, 977

Tong Y., Vasko I. Y., Pulupa M., Mozer F. S., Bale S. D., Artemyev A. V., Krasnoselskikh V., 2019a, ApJL, 870, L6

Tong Y., Vasko I. Y., Artemyev A. V., Bale S. D., Mozer F. S., 2019b, The Astrophysical Journal, 878, 41

Viñas A., Gurgiolo C., Nieves-Chinchilla T., Gary S., Goldstein M., 2010, in AIP Conference Proceedings. pp 265-270, doi:10.1063/1.3395852

Wilson III L. B., et al., 2013, J. Geophys. Res., 118, 5

Yoon P. H., 2017, Reviews of Modern Plasma Physics, 1, 4

Štverák S̆., Trávníček P., Maksimovic M., Marsch E., Fazakerley A. N., Scime E. E., 2008, J. Geophys. Res., 113, A03103

This paper has been typeset from a $\mathrm{T}_{\mathrm{E}} \mathrm{X} / \mathrm{L} \mathrm{T}_{\mathrm{E}} \mathrm{X}$ file prepared by the author. 